HABIBOLLAH NASSIRI, Ph.D. ${ }^{1}$

(Corresponding author)

E-mail: nassiri@sharif.edu

SARA TABATABAIE, Ph.D. Candidate ${ }^{2}$

E-mail: sara.tabatabaie@colorado.edu

SINA SAHEBI, Ph.D. Candidate ${ }^{1}$

E-mail: sina_sahebi@mehr.sharif.ir

${ }^{1}$ School of Civil Engineering, Sharif University

of Technology, Azadi Ave, Tehran, Iran

2 Environmental Studies Program,

University of Colorado, Boulder, Colorado, U.S.
Traffic Management Original Scientific Paper Submitted: 3 Jan. 2016 Accepted: 16 Nov. 2016

\title{
DELAY-BASED PASSENGER CAR EQUIVALENT AT SIGNALIZED INTERSECTIONS IN IRAN
}

\begin{abstract}
Due to their different sizes and operational characteristics, vehicles other than passenger cars have a different influence on traffic operations especially at intersections. The passenger car equivalent (PCE) is the parameter that shows how many passenger cars must be substituted for a specific heavy vehicle to represent its influence on traffic operation. PCE is commonly estimated using headway-based methods that consider the excess headway utilized by heavy vehicles. In this research, the PCE was estimated based on the delay parameter at three signalized intersections in Tehran, Iran. The data collected were traffic volume, travel time for each movement, signalization, and geometric design information. These data were analysed and three different models, one for each intersection, were constructed and calibrated using TRAF-NETSIM simulation software for unsaturated traffic conditions. PCE was estimated under different scenarios and the number of approach movements at each intersection. The results showed that for approaches with only one movement, PCE varies from 1.1 to 1.65. Similarly, for approaches with two and three movements, the PCE varies from 1.07 to 1.99 and from 0.76 to 3.6, respectively. In addition, a general model was developed for predicting PCE for intersections with all of the movements considered. The results obtained from this model showed that the average PCE of 1.5 is similar to the value recommended by the HCM (Highway Capacity Manual) 1985. However, the predicted $P C E$ value of 1.9 for saturated threshold is closer to the PCE value of 2 which was recommended by the HCM 2000 and HCM 2010
\end{abstract}

\section{KEY WORDS}

passenger car equivalent; signalized intersection; delay; simulation;

\section{INTRODUCTION}

A heavy vehicle is defined as a vehicle with gross mass of 4.5 tonnes or more [1, 2]. The proportion of these vehicles in urban street traffic is considerably lower than passenger cars. Notwithstanding this low percentage, the major effects of heavy vehicles on traffic flow have caused them to be seriously studied [3]. Heavy vehicles use more space than passenger cars, their accelerating and decelerating characteristics, and their ability to manoeuvre is less than for passenger cars. In addition, they cause sight distance restrictions for other vehicles in their vicinity.

In the design of a signalized intersection, it is very important to determine the capacity of the intersection, and passenger car equivalent (PCE) estimation is essential to ascertain measures of signalized intersection capacity. The HCM (Highway Capacity Manual) does not consider the differences in operational characteristics of road and traffic streams and uses a constant PCE value. In HCM 1985, the PCE of 1.5 for signalized intersections was assumed. This factor increased to 2 in HCM 1994, 1997 and 2003 [4]. However, since the PCE depends on factors influencing traffic flow parameters [5], setting it at a constant value under different roadway and traffic conditions may not be correct. Therefore, development authorities are developing standards based on regional characteristics [6]. Different regional investigations are necessary to obtain appropriate values of PCE.

Ideally, predictive models in accordance with the characteristics of the region and traffic conditions are required. In this paper, an empirical and analytical study was carried out to determine the PCE of heavy vehicles at signalized intersections in Iran and thereby a predictive linear model proposed the PCE at signalized intersection approaches.

\section{LITERATURE REVIEW}

The PCE concept is the number of passenger cars that are substituted for a truck or bus under a specific traffic situation. Many methods for evaluating this 
factor have been introduced. These methods substantially estimate PCE by comparing one of the parameters related to the level of service such as flow, average speed, average headway in basic flow and mixed flow, and the setting relationships between them [7].

In 1947, Greenshields et al. formulated the PCE value by dividing the average headway of heavy vehicles type over the average headway of passenger cars. This method, called the headway ratio method, is commonly used for measuring PCE at signalized intersections $[5,7]$. Branston and van Zuylen developed a multiple regression method for estimating $P C E$ at signalized intersections in 1978. In this method, vehicle departures are recorded over an arbitrary period during the green light. Within a specific period, the number and type of vehicles departing are recorded. Then, the linear regression model describes the number of passenger cars by the number of vehicles of other classes (the number of each type of vehicles with the exception of passenger cars) as the explanatory variables. The PCE for through movement value achieved by Branston and van Zuylen was 1.59 [8]. After the Branston and van Zuylen's study, the multiple regression analysis method was used to drive PCE factors in many studies [6].

In 1984 Sumner et al. described a method for estimating PCE between signalized intersections. They collected field data on traffic stream and simulated their flow using NETSIM. They estimated the PCE value of 1.6 for heavy trucks [9].

In 2000, Benekohal and Zhao introduced a method that considered induced delay on signalized intersections as the basis of specifying PCE. They named the resulting $P C E, d-P C E$ (i.e. delay-based passenger car equivalent). In this method, $d-P C E$ is estimated according to the following formula [5]:

$$
d-P C E_{i}=1+\frac{\Delta d_{i}}{d_{0}}
$$

where $d-P C E_{i}$ is the passenger car equivalent of vehicle $i, \Delta d_{i}$ is the extra delay caused by the entrance of vehicle $i$, and $d_{0}$ is the basic delay (average delay of passenger cars in the basic flow). Benekohal and Zhao used $d-P C E$ to adjust the ideal saturation flow rate at intersections using $\mathrm{HCM}$. The saturated flow rate of each lane at a signalized intersection is considered to be about 1,900 pcpgpl (passenger car per green signal phase per lane) according to HCM. For evaluating the exact capacity of the lane, this flow must be multiplied by a decreasing factor, FHV, which shows the influence of heavy vehicles on the traffic flow. Equation 2 calculates FHV [7]:

$$
F_{H V}=\frac{1}{1+\sum_{i=1}^{n} P_{H i}\left(P C E_{i}-1\right)}
$$

where $P_{H i}$ is the proportion of heavy vehicles $i$ in traffic flow, $P C E_{i}$ is the passenger car equivalent for vehicle $i$, and $n$ is the number of vehicles other than passenger cars in traffic flow.

In recent years, there have been several studies on estimating regional values of $P C E$. The current literature on regional $P C E$ estimation abounds with examples of PCE at midblock locations $[6,10,11]$ and $P C E$ at intersections [12-14].

In 2009, Saha et al. investigated the value of PCE for through vehicles according to the traffic conditions of Dhaka [12]. They used a headway ratio method for estimating the PCE for cars, auto-rickshaws, mini-buses, and buses. These four vehicle types reflected the actual traffic conditions of Dhaka. They demonstrated that the PCE currently used in Bangladesh significantly differs from the PCE achieved from their study. They estimated 1.00, 0.86, 1.42, and 2.16 for PCE values of cars, auto-rickshaws, mini-buses, and buses, respectively [12].

In 2014, Yahya and Sarraj estimated PCE for medium trucks, heavy trucks, and animal-driven carts in mixed traffic conditions at signalized intersections in Gaza [14]. The study was conducted in the headway ratio method, with data being gathered via digital video camera at three signalized intersections. The estimated PCEs for medium trucks, heavy trucks, and animal-driven carts were $1.43,2.23$, and 1.51, respectively. They compared the estimated PCEs with the PCE currently used in the UK and India. These comparison findings showed contrariety of PCE value for Gaza, UK, and India [14].

A similar study was done in Kumasi city, Ghana in 2014 [13]. In this study, Obiri-Yeboah et al. analysed eleven pre-timed signalized intersections to assess the influence of roadside friction on the value of PCE. They found PCE values were higher at intersections with roadside friction than the ones without such friction. Obiri-Yeboah et al. showed the PCE value at intersection in Kumasi was larger than the value prevalently used [13]. The results of all current studies supported the claim that the values of PCE are different for each country due to differences in driving behaviours, available transport facilities, and so on.

\section{METHODOLOGY}

The aim of this paper is to develop a predictive model for estimating $d-P C E$ in Iran. According to the Benekohal and Zhao's study [5], the volumes and heavy vehicle percentage have been identified as major factors affecting $d-P C E$. Therefore, to attain a $d-P C E$ predictive model, samples of intersection delays varying by traffic volume and heavy vehicle percentage are needed. The samples establishing a general relationship between $d-P C E$, traffic volume and heavy vehicle 
percentage, must be generated by a calibrated simulation software. For the sake of simulation, it might be convincingly argued that the broad range of field data does not support model prediction. To achieve the proper conditions that were not obtained from the field data, it was necessary to simulate the intersections under different circumstances. Thus, the results could be generalized to other traffic conditions.

Another argument that can be advanced to support the simulation idea is that field data include many factors affecting the measuring of the pure impact of heavy vehicles stated by $d-P C E$.

This paper puts forward a predictive model of $d-P C E$ that used samples generated by calibrated intersection simulation software. This simulation was done using two groups of data; simulation field data and calibration field data. The simulation field data were used to define the traffic conditions and geometric characteristics of intersections. The calibration field data measured the intersection delay parameter used to validate the simulation process. The detailed data gathering procedure is described in the following section.

The PCE for saturated and under-saturation traffic conditions can be estimated differently and the PCE factors should not be used to conduct analyses for all traffic conditions similarly [15].This study was restricted to under-saturated traffic conditions and a limited range of heavy vehicle percentage. Further research in this area may include PCE estimation on saturated traffic conditions and a broader range of heavy vehicle percentage.

\section{DATA COLLECTION}

The field data for the study were collected in Tehran. The data collection process was carried out during weekdays in November, between 7:00 a.m. and 10:00 a.m. (peak hours of traffic volume in Tehran). Fourteen approaches were surveyed at five intersections for field data collection.

These approaches passed through two strainers in data reduction procedure. In these two steps the intersections and approaches were selected based on criteria compiled from research conducted in Benekohal and Zhao's study [5]. In the first step, the intersections were chosen based on the features listed below:

- All the intersections had pre-timed signal timing during the time of the survey;

- Flows at intersections were sustained at the saturation flow rate level;

- The selected intersections represented the typical ones with common geometric design conditions;

- These intersections were chosen from different locations throughout the city of Tehran to represent all the city intersections.

In accordance to the provided criteria, four intersections were chosen as preliminary data survey fields. It should be noted that in some filtered intersections, the resultant approach was not surveyed because of heavy traffic and over-saturated conditions. Appropriate approaches to the selected intersections were chosen in the second step of data reduction. Surveyed approaches were ranked based on the following conditions:

- The approach was under-saturated for at least 1.5 hours of the 3 hours surveying;

- These intersections were controlled in a pre-timed signal manner;

- Under the current signalization, all approaches of different movements were done in the same phase and with the least conflict with other approaches;

- The effects of external factors, such as stop and go manoeuvres by taxis and congestion at adjacent intersections that resulted in a queue and increased delay was minimal on the approach delay;

- Intersections represented common intersection types from the viewpoint of location, volume, and percentage of heavy vehicles;

- The approach movement delays did not fluctuate substantially from one interval to another.

Finally, three approaches were selected from the twelve preliminary approaches for more studies, namely, Ostad Hassan Banna, Shariati (South to North), and Mojahedin (West to East). Table 1 illustrates some features of these approaches. This table shows direction, type of movement, operational classification, and lane width of the selected surveyed approaches.

For modelling the intersections and obtaining suitable results, various data had to be collected. As mentioned in the methodology section, the necessary field

Table 1 - Selected surveyed approaches characteristics

\begin{tabular}{||c|c|c|c|c|c|c|c||}
\hline $\begin{array}{c}\text { Approach } \\
\text { Name }\end{array}$ & $\begin{array}{c}\text { Minimum Vol- } \\
\text { ume [veh/hr] }\end{array}$ & $\begin{array}{c}\text { Maximum Vol- } \\
\text { ume [veh/hr] }\end{array}$ & $\begin{array}{c}\text { Direction of } \\
\text { Movement }\end{array}$ & $\begin{array}{c}\text { Type of } \\
\text { Movement }\end{array}$ & $\begin{array}{c}\text { Approach } \\
\text { Classification }\end{array}$ & Width [m] $)$ & $\begin{array}{c}\text { Volume } \\
\text { Percentage }\end{array}$ \\
\hline \hline Mojahedin & 100 & 1200 & West to East & Through & Major Arterial & 7.4 & $100 \%$ \\
\hline \multirow{2}{*}{ Shariati } & 100 & 1600 & South to East & Right Turn & Major Arterial & 9.3 & $15 \%$ \\
\cline { 4 - 9 } & & South to Noth & Through & Major Arterial & 9.3 & $85 \%$ \\
\hline $\begin{array}{c}\text { Ostad } \\
\text { Hassan } \\
\text { Banna }\end{array}$ & 100 & \multirow{2}{*}{1100} & North to West & Right Turn & Major Arterial & 11.7 & $35 \%$ \\
\cline { 4 - 9 } & & North to South & Through & Major Arterial & 11.7 & $45 \%$ \\
\cline { 4 - 9 } & & North to East & Left Turn & Major Arterial & 11.7 & $20 \%$ \\
\hline
\end{tabular}


data included two groups of data: simulation field data and calibration field data.

The simulation field data determined the traffic conditions and geometric characteristics of the intersectionsforfeedingthesimulationsoftware. Theseincluded volume for each approach movement classified by vehicle type, geometric data of each intersection, and signalling data.

It should be noted that without such calibration and validation, simulation software could not be used for the intersection simulation [5]. There was, therefore, an essential need for the calibration field data. The delay of each approach movement in five-minute intervals was surveyed as a parameter that could validate the simulation process. The travel time method was used to collect the delay data at the intersections [7].

The travel time method needed four surveyors. The first two surveyors stood at the end of each approach queue line. These surveyors wrote down the last three digits of passenger cars and the exact time the passenger cars passed them. After matching the similar license plates, aside from identifying the vehicles route, the travel delay for each passenger car was obtained by subtracting free flow travel time from the obtained travel time (i.e. the last time a passenger car was observed minus the first time it was recorded). The resulted delay values were averaged in five-minute intervals for each movement (i.e. left turn, through movement, right turn) [16].

\section{MODELLING SELECTED INTERSECTIONS IN TRAF-NETSIM}

The foregoing discussion in the methodology section implies that the use of a simulation model is vital to cover a broad range of traffic conditions. In this research TRAF-NETSIM, the simulation software, was used to generalize field data to other traffic conditions. This software is a microscopic simulation tool that simulates operation of vehicles in an urban network using the discrete event simulation method [17].

After the proper approaches had been specified, the intersections that included these approaches were modelled in TRAF-NETSIM. Since then the t-statistic test showed some differences between the real and simulated data. Therefore, the models had to be calibrated and some behavioural parameters were adjusted for conditions specific to Iran. Abedini discusses the challenges and modifications of calibration of the TRAF-NETSIM for Iran's signalized intersections [18]. His sensitivity analysis showed that free flow speed, start-up lost time, and discharge headway are variables that must be modified in the calibration process [18].
In the process of modelling the intersections in the simulation software, the following issues were considered:

- Arrival type 3 was considered for selected approaches.

- Two series of random data were established, one of which was related to the behavioural characteristics of the drivers, and the other is related to the random distribution of time-dependent events, such as the location of the obstruction on a lane.

- Given that, it was possible that over a period of 5 minutes no green phase happened at the entrance, volume of entry to the software was considered as the average of volume of 5 minutes before, current, and after.

- To compare different strategies of a network, it is desirable that differences exist solely due to changes in network status to be tested. In such a situation, the same values should be selected for the cores. Such a technique is known as Common Random Number technique (CRN).

Calibration of TRAF-NETSIM for our study was evaluated by measuring the sensitivity of delay to various default parameters. Then, the default values of the effective parameters were changed to calibrate the models. The default parameters of free flow speed, start-up lost time, and discharge were determined through survey data. Other parameters such as short time events were changed in a way to satisfy the t-statistic test for averaging the movement delay. In addition, vehicle characteristics were calibrated to satisfy Iran's standards.

Although the geometric characteristics, signal phase plans, and movement shares at approaches were defined in TRAF-NETSIM, the intersection simulation models were not finalized unless their validity could be verified. Delay is one of the most important parameters to measure intersection effectiveness. Furthermore, this parameter is affected by almost all other traffic parameters. Therefore, movement average delay was determined as a criterion to validate the model.

For each approach, the average delay was estimated for different volumes from $100 \mathrm{veh} / \mathrm{hr}$ to saturation flow level by 50 veh/hr increments (the saturation flow rate for each intersection has been reported in Table 1). Also different percentages of heavy vehicles that varied from 2 to 26 percent by 2 percent increments for each volume. The delay for each case resulted from using CRN. CRN is a method used for simulation studies where the same random numbers are used to simulate both alternative system designs [19].

\section{RESULTS AND ANALYSIS}

After the validity of each intersection model had been proven they were substituted for the real 
intersection. The simulation outputs showed the delay of each movement at the approaches. These outputs were transferred to the approach delay by Equation 3. The $d$-PCE was estimated by substituting the approach delay into Equation 4. Equation 3 and Equation 4 are shown below:

$d_{m}=V_{m} \cdot \sum_{i=1}^{n} d_{m i} \cdot P_{i m}$

$$
d-P C E=1+\frac{d_{m}-d_{0}}{P_{H} \cdot d_{0}}
$$

where $d_{m}$ is the average delay of approach $m, v_{m}$ is traffic volume of approach $m, d_{m i}$ is the delay of movement $i$ in approach $m, P_{i m}$ is the percentage of movement $i$ in approach $m, d_{0}$ is the basic delay, and $P_{H i}$ is the proportion of heavy vehicles $i$ in traffic flow.

In Equation 4 we needed to calculate the basic delay $\left(d_{0}\right)$. The basic delay is the average delay of passenger cars in the basic flow or flow with zero percent heavy vehicles. The simulation models were run at zero percent of heavy vehicles and considered the volume needed to obtain the basic delays at each approach. The zero percent heavy vehicle condition was not given in the field data, so the basic delay estimation supported the claim that the use of simulation in this study was inevitable.

Changes from one approach to another showed that the evaluated $d$-PCE parameter for the surveyed conditions was not constant. In addition, $d$-PCE varied significantly for different volumes and heavy vehicle percentages. Therefore, it was necessary to study the sensitivity of $d-P C E$ to volume, heavy vehicle percentage and the number of movements of each approach. To do this the results of models run in different situations had to be compared. To evaluate how $d-P C E$ varied by volume and percentage of heavy vehicles, $d$-PCE diagrams versus different volumes were plotted at three constant percentages in Figure 1. In addition, $d-P C E$ diagrams versus different heavy vehicle percentages for constant volumes in each approach were plotted in Figure 2. These diagrams show:

- In most cases $d$-PCE had little sensitivity to the percentage of heavy vehicles.

- $d$-PCE versus volume had an ascending trend, which seemed smoother in the upper bound.

- The results were sporadic at lower volumes and as the volume increased, the results became more tangible.

The data yielded by this paper provide strong evidence that shows more operation of an isolating heavy vehicle effect on the traffic stream. This finding along with the PCE value pattern of HCM and Benekohal's ascertainment [5] lend support to the claim that the maximum impact of a single heavy vehicle occurs when it is relatively isolated in the traffic stream. Along similar lines, McShane argues that as the flow of heavy vehicles increases, they begin to form their own platoons within which they can operate more efficiently

[7].

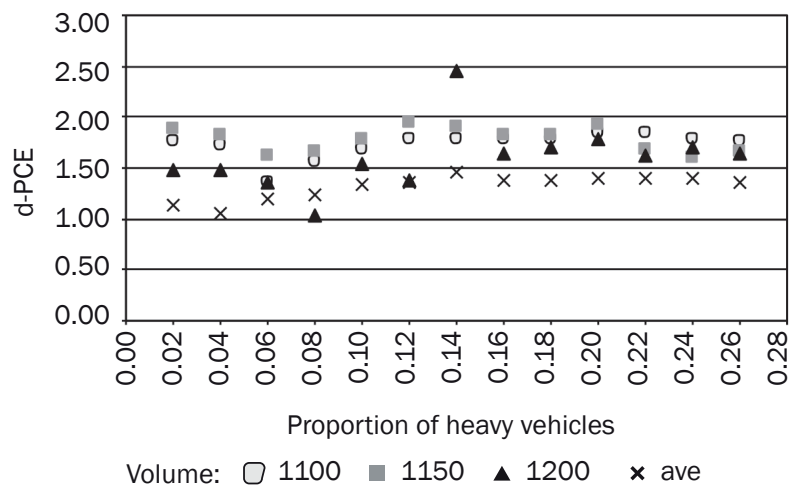

Figure 1 - d-PCE values for different heavy vehicle percentages at a given traffic volume (veh/hr)

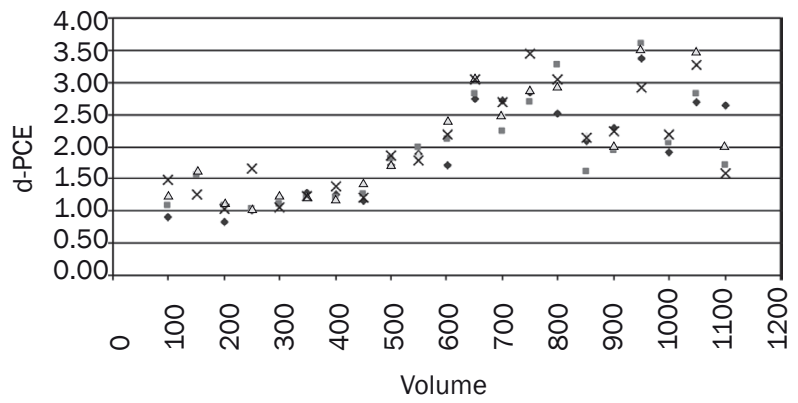

Proportion of heavy vehicles: $\bullet 0.18 \square 0.2 \Delta 0.22 \times 0.24$

Figure 2 - d-PCE values for different traffic volume (veh/hr) at given heavy vehicle percentages

Figure 2 shows for a given percentage of trucks $d-P C E$ increased with the traffic volume. The positive correlation of traffic volume and $d-P C E$ that was found in this study is prominent in the literature on PCE estimation. However, from the data in Figure 2 it is apparent that the trends of results were flattened at higher traffic volumes. The underlying argument in favour of this phenomenon is the manoeuvrability of heavy vehicles. As mentioned before, one of the primary reasons for more displacing of heavy vehicles is that manoeuvrability of heavy vehicles is lower than of passenger cars. This ability, by increasing the traffic volume, is reduced at the higher traffic volume and the manoeuvrability reduction decreases the rate of the increasing $d$-PCE. Therefore, there is a flattening at higher traffic volumes in Figure 2. A closer look at the data indicates that the average $d-P C E$ increased with approach movements. This evidence could be legitimized by considering the reasons for the observed flattening. Since the volume and the percentage of heavy vehicles affect $d-P C E$, this trend must also be viewed analytically in order to have 
Table 2 - d-PCE predictive models

\begin{tabular}{|c|c|c|c|c||}
\hline Number of movements & $d-P C E$ formulas & $\begin{array}{c}d-P C E \text { at average } \\
\text { volume } / w\end{array}$ & $\begin{array}{c}\text { t-statistic of } \\
\text { volume } / w\end{array}$ & $R^{2}$ \\
\hline \hline 1 & $d-P C E=1.105+3.34 \cdot 10^{-3} \cdot($ volume $/ w)$ & 1.35 & 11.31 & 0.33 \\
\hline 2 & $d-P C E=1.03+5.48 \cdot 10^{-3} \cdot($ volume $/ w)$ & 1.44 & 19.98 & 0.54 \\
\hline 3 & $d-P C E=0.579+1.83 \cdot 10^{-3} \cdot($ volume $/ w)$ & 1.95 & 16.8 & 0.53 \\
\hline General Condition & $d-P C E=1.011+6.43 \cdot 10^{-3} \cdot($ volume $/ w)$ & 1.5 & 14.3 & 0.46 \\
\hline
\end{tabular}

mathematical models for estimating $d-P C E$ in different conditions.

Because of the considerable effect of a number of movements on $d-P C E$, three linear regression models were developed separately for each number of movements (i.e. 1, 2, and 3). However, an integrated model for all the number of movements could facilitate the use of predictive $d$-PCE models. Therefore, four models were developed in the modelling process. Several mathematical forms of variables (i.e. logarithmic and powered form of traffic volume and heavy vehicle percentage inverse) examined to fit the linear regression model.

In Iran, the drivers do not mainly drive between the lanes and a three-lane way may be used in four lanes. For this reason, using the number referring to road width as a normalizing variable is preferred to the number of lanes. The final models are illustrated in Table 2. The " $w$ " shown in Table 2 is the width of the riding way.

The $d$-PCE calculation rests on the assumption that the growth of heavy vehicle percentage entailed increasing approach delay and a d-PCE value of less than one for non-zero heavy vehicle percentage is not meaningful. Therefore, a $d-P C E$ value of one must be considered the maximum and the estimation value of the simulation model. In other words, $d-P C E=\operatorname{Max}(1$, estimation of model).

To find models that can predict $d-P C E$, the patterns of evidences in Figures 1 and 2 were noted and regression models were developed. The t-statistics showed the parameters were estimated at a high significant level ( $p$-value<0.01). These models show volume has a direct and positive effect on $d-P C E$.

In justifying the lack of significance of the number of heavy vehicles in the presented model, it must be said that the volume of traffic is a very important factor that is significantly correlated with the volume of heavy vehicles, too. It seems that this high correlation led to the fact that in the presence of traffic volume variable, the variable of the number of heavy vehicles became insignificant. However, from a practical standpoint, having a relationship based on traffic volume to calculate $P C E$ is much more efficient than the relationship based on the number of heavy vehicles, since the traffic of heavy vehicles at the intersection occurs with a low rate and high variance.
This finding is supported by most research on PCE estimation. In this research, to generalized use of the predictive model, the volume was replaced with volume per lane width. All of these models showed that the percentage of heavy vehicles had no significant effect on $d-P C E$.

In Table 2, the d-PCE at 75 vehicles per hour per meter, called $d-P C E$ at average volume $/ w$, for each model was reported. It can be seen that, when the number of movements considered at an approach increased, the $d$-PCE at the average volume/w became larger. This is because of more ability of heavy vehicle manoeuvre at approaches with higher movement potential. The $d-P C E$ estimated values were not constant, but varied from 1.07 to 2.07 for general condition and from 1.15 to 1.65 for one movement at approach. Similarly, for approaches with two and three movements, the PCE varies from 1.07 to 1.99 and from 0.76 to 3.6, respectively. The finding values were compared with the value of $P C E$ suggested by HCM.

\section{CONCLUSION}

This study draws on research conducted by Benekohal and Zhao [5], to develop a predictive model of PCE at Iran's signalized intersections. The TRAF-NETSIM simulation model was used to cover variant traffic conditions. This model was calibrated based on the field data at Iran's intersections. The simulations result analyses showed that $d-P C E$ is sensitive to traffic volume and number of movements at the intersection approach. In addition, investigating different models showed that linear regression models provided a better fit to the d-PCE data versus traffic volume compared to the other models. Therefore, four linear regression models were developed based on the traffic volume for each number of movements as predictive models. Models showed an increase in traffic volume resulting in an increase in $d-P C E$, as expected. The higher number of movements resulted in a steeper slope of $d-P C E$ versus volume.

The results obtained from this model show that the average PCE of 1.5 is similar to the value recommended by HCM 1985. However, the predicted PCE value of 1.9 for saturated threshold is closer to the PCE value of 2 , recommended by HCM 2000. Therefore, the 
current research on PCE estimation does not support the $\mathrm{HCM}$ view and the constant PCE recommended in HCM overestimated the PCE for Iran's signalized intersections. The foregoing discussion implies that since PCE depends on factors influencing the traffic flow parameters, setting it at a constant value under different roadway and traffic conditions does not have to be correct.

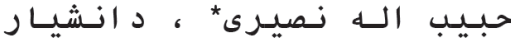

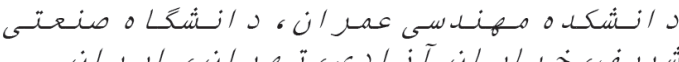

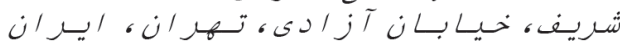

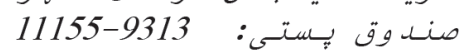

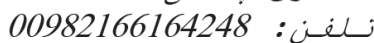

$$
\begin{aligned}
& \text { فـسن: } 00982166014828 \\
& \text { nassiri@sharif.edu }
\end{aligned}
$$

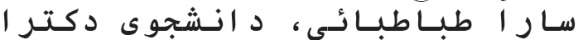

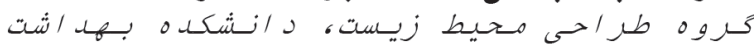

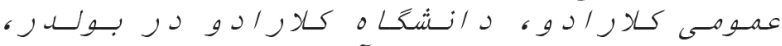

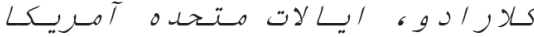

$$
\begin{aligned}
& \text { تـلنفن: } 0017204128989 \\
& \text { sara.tabatabaie@colorado.edu }
\end{aligned}
$$

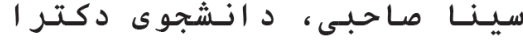

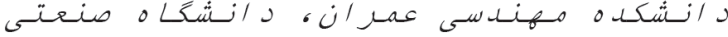

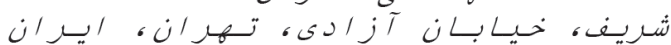

$$
\begin{aligned}
& \text { 00989112542128 } \\
& \text { sinasahebi@mehr.sharif.ir }
\end{aligned}
$$

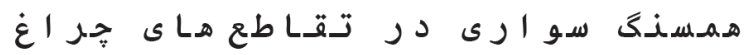

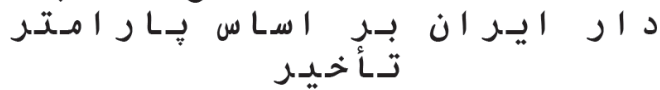

$$
\text { جــــــ }
$$

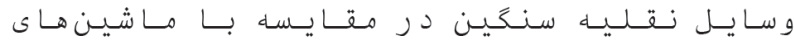

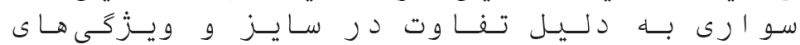

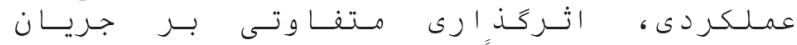

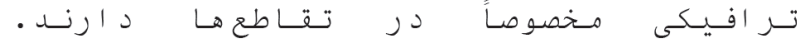

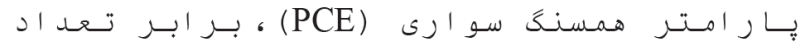

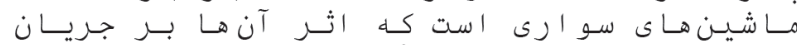

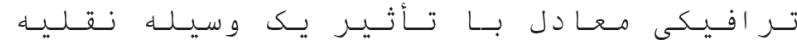

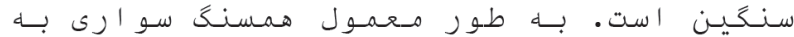

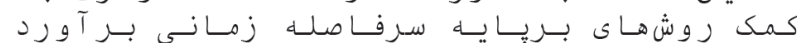

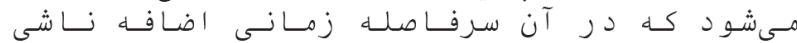

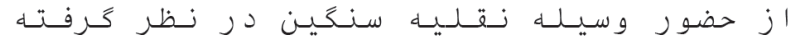

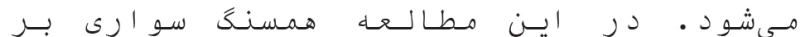

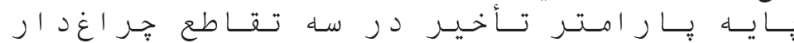

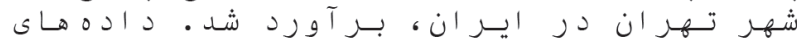

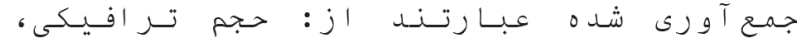

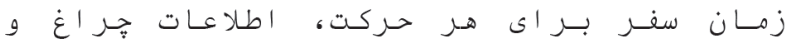

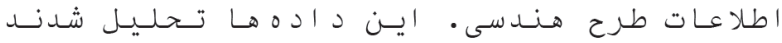

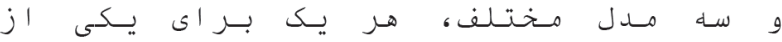

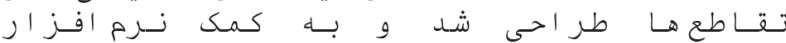

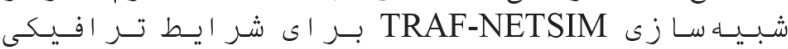

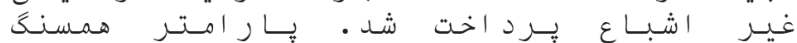

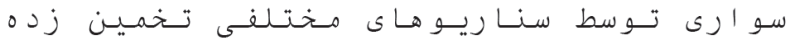

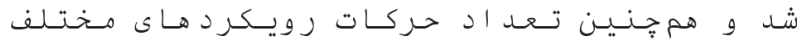

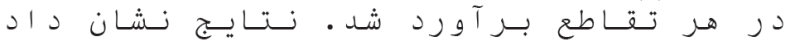

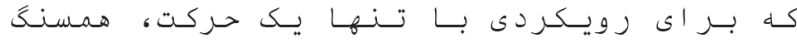

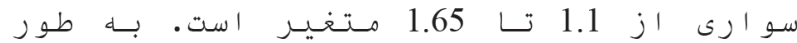

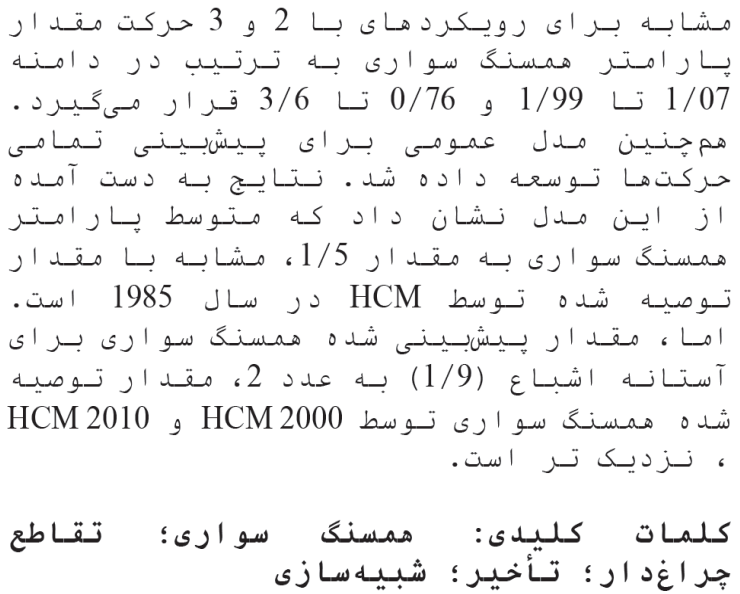

\section{REFERENCES}

[1] Knipling R, Waller P, Peck R, Pfefer R, Neuman T, Slack K, et al. NCHRP Report 500: Guidance for Implementation of the AASHTO Strategic Highway Safety Plan, Volume 13: A Guide for Reducing Collisions Involving Heavy Trucks. Transportation Research Board, Washington, DC; 2004.

[2] Brodie L, Lyndal B, Elias IJ. Heavy vehicle driver fatalities: Learning's from fatal road crash investigations in Victoria. Accident Analysis \& Prevention. 2009;41(3):557-64.

[3] Al-Kaisy AF, Hall FL, Reisman ES. Developing Passenger Car Equivalents for Heavy Vehicles on Freeways During Queue Discharge Flow. Transportation Research Part A: Policy and Practice. 2002;36(8):725-42.

[4] Kockelman KM, Shabih RA. Effect of Vehicle Type on The Capacity of Signalized Intersections: The Case of Light-Duty Trucks. Journal of Transportation Engineering. 1999;126(6):506-12.

[5] Benekohal RF, Zhao W. Delay-based passenger car equivalents for trucks at signalized intersections. Transportation Research Part A: Policy and Practice. 2000;34(6):437-57.

[6] Adnan M. Passenger Car Equivalent Factors in Heterogeneous Traffic Environment-are We Using the Right Numbers? Procedia Engineering. 2014;77:106-13.

[7] McShane WR, Roess RP, Prassas ES. Traffic Engineering. New Jersey: Prentice Hall; 1998.

[8] Branston D, van Zuylen $H$. The estimation of saturation flow, effective green time and passenger car equivalents at traffic signals by multiple linear regression. Transportation Research. 1978;12(1):47-53.

[9] Sumner R, Hill D, Shapiro S. Segment passenger car equivalent values for cost allocation on urban arterial roads. Transportation Research Part A: General. 1984;18(5):399-406.

[10] Ingle A. Development of Passenger Car Equivalents for Basic Freeway Segments. Blacksburg, Viginia: Virginia Polytechnic Institute and State University; 2004. 
[11] Anwaar A, Van Boxel D, Volovski M, Anastasopoulos PC, Labi S, Sinha KC. Using Lagging Headways to Estimate Passenger Car Equivalents on Basic Freeway Sections. Journal of Transportation of the Institute of Transportation Engineers. 2011;2(1):5-17.

[12] Saha P, Hossain QS, Mahmud HMI, Islam MZ. Passenger Car Equivalent (PCE) of Through Vehicles at Signalized Intersections in Dhaka Metropolitan City, Bangladesh. IATSS Research. 2009;33(2):99-104.

[13] Obiri-Yeboah AA, Tuffour YA, Salifu M. Passenger Car Equivalents for Vehicles at Signalized Intersections within the Kumasi Metropolis in Ghana. IOSR Journal of Engineering. 2014;4(4):24-9.

[14] Sarraj YR. Passenger Car Equivalents at Signalized Intersections for Heavy and Medium Trucks and Animal Driven Carts in Gaza, Palestine. International Journal of Emerging Technology and Advanced Engineering 2014;4(2):80-8.

[15] Al-Kaisy A, Jung Y, Rakha H. Developing passenger car equivalency factors for heavy vehicles during congestion. Journal of transportation engineering. 2005;131(7):514-23.

[16] Richardson AJ, Ampt ES, Meyburg AH. Survey Methods for Transport Planning: Eucalyptus Press; 1995.

[17] Rathi AK, Santiago AJ. Urban Network Traffic Simulations: TRAF-NETSIM Program. Journal of transportation engineering. 1990;116(6):734-43.

[18] Abedini M. TRAF-NETSIM Calibration for Iran Signalized Intersections. Tehran, Iran: University of Tehran; 1999.

[19] Banks J. Discrete-event System Simulation: Prentice Hall; 2010. 